\title{
High-Definition Optical Coherence Tomography for the in vivo Detection of Demodex Mites
}

\author{
T. Maier E. Sattler M. Braun-Falco T. Ruzicka C. Berking \\ Department of Dermatology and Allergology, Ludwig-Maximilian University of Munich, Munich, Germany
}

\section{Key Words}

Skin disease $\cdot$ Demodex mites $\cdot$ High-definition optical coherence tomography

\begin{abstract}
Background: Demodex mites are involved in different skin diseases and are commonly detected by skin scrape tests or superficial biopsies. A new high-definition optical coherence tomography (HD-OCT) with high lateral and axial resolution in a horizontal (en-face) and vertical (slice) imaging mode might offer the possibility of noninvasive and fast in vivo examination of demodex mites. Methods: Twenty patients with demodex-related skin diseases and 20 age- and gender-matched healthy controls were examined by HDOCT. Mites per follicle and follicles per field of view were counted and compared to skin scrape tests. Results: HDOCT images depicted mites in the en-face mode as bright round dots in groups of 3-5 mites per hair follicle. In the patients with demodex-related disease, a mean number of 3.4 mites per follicle were detected with a mean number of 2.9 infested follicles per area of view compared to a mean of 0.6 mites in 0.4 infested follicles in the controls. The skin scrape tests were negative in $21 \%$ of the patients. Conclusion: The innovative HD-OCT enables fast and noninvasive in vivo recognition of demodex mites and might become a useful tool in the diagnosis and treatment monitoring of demodex-related skin diseases.

Copyright $\odot 2012$ S. Karger AG, Basel
\end{abstract}

\section{Introduction}

Demodex mites have been identified in different inflammatory skin diseases of the face such as rosacea, demodex folliculitis and demodex-aggravated perioral dermatitis [1-5]. The mites are believed to have an aggravating potential in preexisting dermatoses such as rosacea $[6,7]$. Although they are occasionally found in healthy facial skin, the pathogenic potential seems to increase with the amount of mites present in the follicles [2]. In this context, the quantification of Demodex folliculorum might be of importance.

Conventionally, demodex mites are diagnosed by invasive or semi-invasive tests like skin scrapes, superficial skin biopsies or the examination of secretions of sebaceous glands by light microscopy $[8,9]$. Besides the invasiveness of these methods, it is not possible to evaluate the mites in vivo in their microenvironment and to quantify them per follicle. Thus, new imaging techniques might be beneficial in the detection and quantification of demodex mites.

Optical coherence tomography (OCT) was introduced into dermatological diagnostics of human skin in the 1990s [10]. Since then, it has been applied in different experimental and clinical settings in dermatology [11]. Cross-sectional images of the skin are produced comparable to the ultrasound technique but near-infrared light is used instead of ultrasound waves. The principle of OCT is based on low-coherence interferometry, in which the

\section{KARGER \\ Fax +4161306 1234 \\ E-Mail karger@karger.ch}

www.karger.com (c) 2012 S. Karger AG, Basel

$1018-8665 / 12 / 2253-0271 \$ 38.00 / 0$

Accessible online at:

www.karger.com/drm
Tanja Maier, MD

Department of Dermatology, Ludwig-Maximilian University

Frauenlobstrasse 9-11

DE-80337 Munich (Germany)

E-Mail Tanja.Maier@med.uni-muenchen.de 
light is divided into a reference and a sample arm and is sent into the tissue. The difference between the two arms generates an interference pattern which can be transformed into cross-sectional images. There are different commercially available OCT devices which offer a lateral and axial resolution of about $5-15 \mu \mathrm{m}$ at best. The crosssectional (B-scan) mode is the conventional imaging mode in OCT but there are devices which offer the possibility of constructing C-scans (en-face), although not in a real-time manner. New technologies have been developed such as the so-called full-field OCT, which provides 3-dimensional images of biological tissues and has recently been applied mainly in an ex vivo setting [12]. Another innovative technique is the high-definition (HD)-OCT which offers a high lateral and axial resolution of $3 \mu \mathrm{m}$ and a real-time en-face imaging mode (C-scan). This has already been evaluated in the in vivo diagnostics of nonmelanoma skin cancer $[13,14]$. In this study, HD-OCT is used for the detection of demodex mites in patients with demodex-related skin eruptions of the face. The clinical interest in this study is mainly the immediate, bedside visualization of demodex mites in comparison to time-consuming laboratory tests such as skin scrape tests.

Conventional OCT has been applied previously in different skin diseases, i.e. in the diagnostics of nonmelanoma skin cancer, for the measurement of tumor thickness of melanocytic lesions, for the evaluation of epidermal changes after different topical treatments and in the assessment of psoriatic nail disorders [13, 15-19].

However, imaging of demodex mites by OCT has not been described before. Therefore, the purpose of this study was to evaluate HD-OCT for the noninvasive in vivo detection and quantification of demodex mites in patients with the clinical diagnosis of demodex-related diseases in comparison with healthy controls and with the results of conventional skin scrapes.

\section{Patients and Methods}

In this prospective pilot study, we examined 20 patients (10 females and 10 males) aged between 34 and 80 years with the clinical diagnosis, after given written informed consent, of rosacea, demodex folliculitis and demodex-aggravated perioral dermatitis by HD-OCT. The HD-OCT imaging was performed at the site with the most prominent clinical manifestation (the cheek in 16 patients, the chin in 3 patients and the forehead in 1 patient); for 20 age- and gender-matched healthy controls, the corresponding site was imaged. The 20 examined patients consisted of patients with rosacea grade $2(n=17)$, demodex folliculitis $(n=2)$ and demodexaggravated perioral dermatitis $(\mathrm{n}=1)$. The investigational site was scanned automatically with multiple en-face images at steps of
$3 \mu \mathrm{m}$ to a maximum depth in the field of view of $1.5 \times 1.8 \mathrm{~mm}$. In all patients, the number of visible hair follicles was counted within the field of view in the en-face mode (follicles/field). Hair follicles displayed as centrally dark round-to-oval structures with a grey surrounding rim in the en-face HD-OCT image (fig. 1). The number of follicles with demodex mites within the field of view (infested follicles) was documented and the number of mites within the infested follicles (mites/follicle) was counted.

In 1 patient (No. 6) with demodex folliculitis, an additional biopsy of the lesional skin on the cheek was performed (fig. 2).

\section{HD-OCT}

OCT was performed using a commercially available full-field HD-OCT system (Skintell ${ }^{\circledR}$, AGFA HealthCare, Belgium). The Skintell system is based on the principle of a 'time domain' OCT system including dynamic focus tracking with the light source of a halogen lamp with a Gaussian filter and an ultra-high bandwidth centered at $1300 \mathrm{~nm}$ (infrared light). According to the manufacturer's instruction, the dynamic focus tracking system implies that the position of the coherence gate and the position of the focal plane are at the same depth location, which results in a highresolution image at all depths [13]. Skintell allows a resolution of $3 \mu \mathrm{m}$ in all 3 dimensions and a penetration depth of about $750 \mu \mathrm{m}$ in the skin according to the manufacturer's instruction.
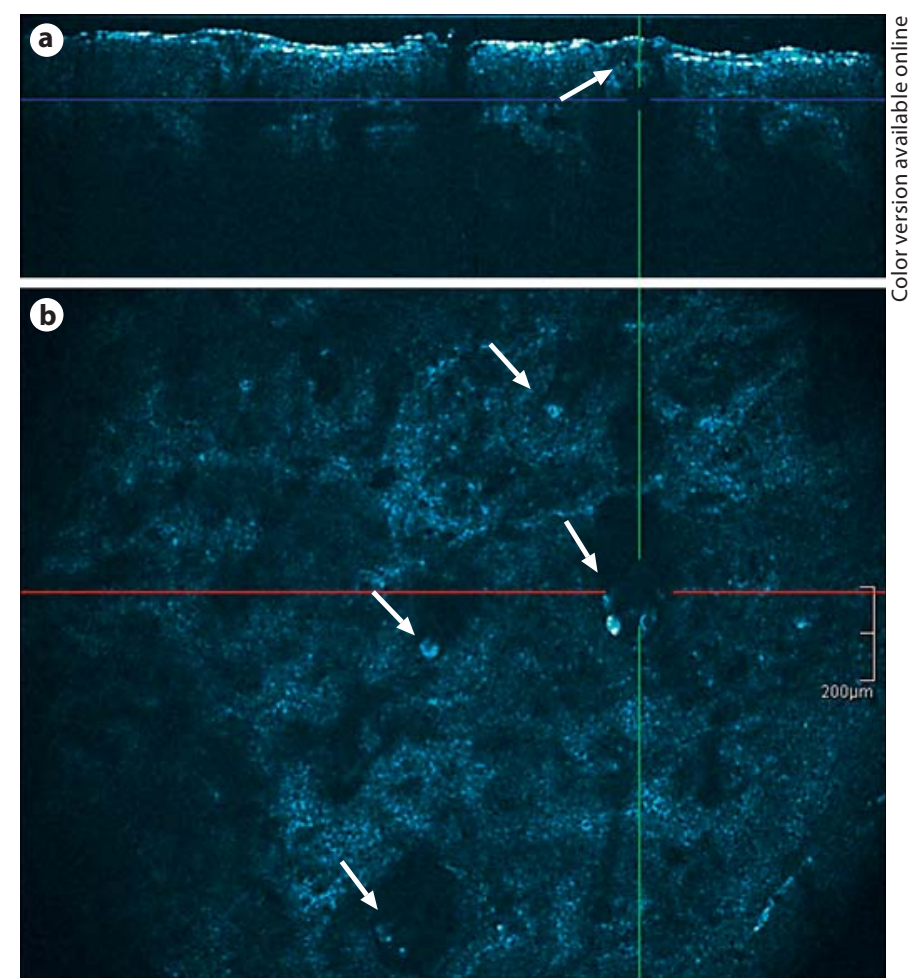

Fig. 1. HD-OCT imaging of facial skin of patient No. 3 (male) showing at least 4 round bright dots at the margins of the dark hair follicle in the en-face mode (b, arrows). Surrounding follicles also contain white dots consistent with an infestation of mites (arrows). In the HD-OCT slice mode (a), only bright white undefined structures are displayed in the follicle. 
The HD-OCT system provides 2 different modes: real-time (B-scan) and en-face (C-scan) and additionally allows fast capture of a 3-dimensional tomogram. The OCT probe is applied directly to the skin with an optical gel (Skintell ${ }^{\circledR}$ optical gel, AGFA HealthCare) as coupling medium. The field of view in the en-face mode is $1.8 \times 1.5 \mathrm{~mm}$.

The clinically suspicious lesions were systematically evaluated by HD-OCT in the slice and en-face mode and images were recorded. The lesions were examined by an experienced investigator (T.M.). The slice mode image is immediately present when applying the probe to the skin. The generation of an en-face image takes less than $1 \mathrm{~min}$ for one image as recorded with an electronic time clock. Including the assessment of patient data and examination site, the whole working process takes less than $5 \mathrm{~min}$.

\section{Skin Scrape Test}

Skin scrape tests were performed by scratching slightly over the lesional skin with a scalpel to obtain superficial skin particles and sebaceous material for evaluation. The material was routinely evaluated on a glass slide under a light microscope by experienced laboratory staff and the result was documented.

\section{Statistical Analysis}

Descriptive statistics were performed to evaluate sensitivity and specificity for HD-OCT detection of demodex mites and $p$ values were determined using the $\chi^{2}$ and the Fisher's exact test. Statistical analysis was performed using the statistical package SPSS (Superior Performance Software System, Munich, Germany). A p value of $<0.05$ was regarded as significant.

\section{Results}

\section{Detection and Quantification of Demodex Mites with HD-OCT}

Demodex mites were detected by HD-OCT in the enface mode as bright round dots in groups of 3-5 dots per hair follicle (fig. 1). The mites were located in the superficial part of the hair follicle. In the slice mode, the detection of mites was not definitely possible. Although in correlation with the findings in the en-face mode, a bright filling with high refractivity was detectable in the miteinfested hair follicles in the slice mode; this is possibly related to sebaceous or horny material (fig. 1). The mites in the follicles of male patients presented clearer and larger than in female patients (fig. 1, 2).

The mean number of hair follicles per imaging field was 12.8 (range 10-16) in the demodex patients compared to 11.7 (range 7-16) in the healthy controls (table 1).

The mean number of infested follicles by demodex mites per imaging field was 2.9 (range 1-7) in the patients with skin eruptions, while in the age- and gender-matched healthy controls the mean number of infested follicles was 0.4 (range $0-1)$ (table 1 ).
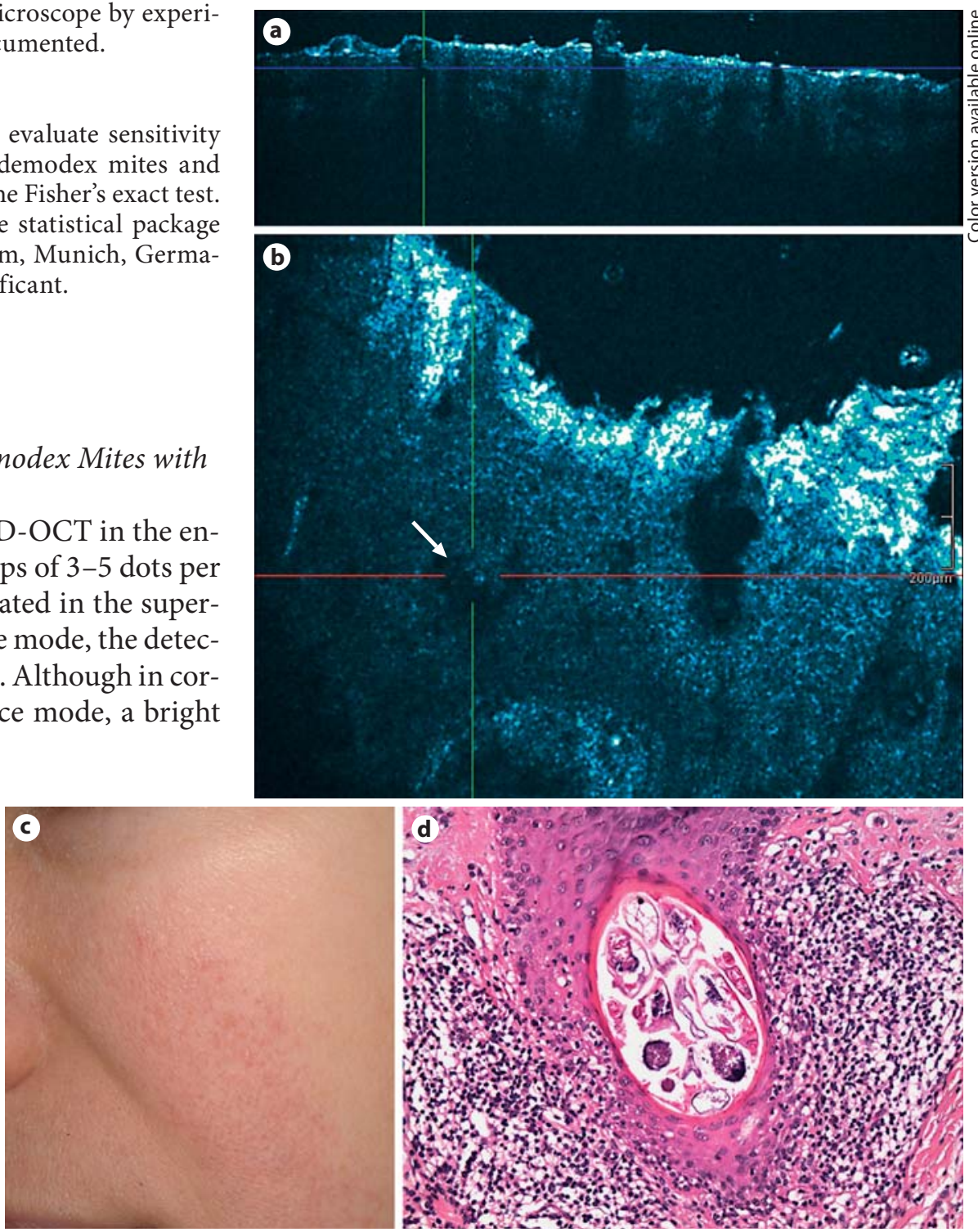

Fig. 2. HD-OCT imaging of patient No. 6 with demodex folliculitis (c) showing infestation of a follicle with at least 2 demodex mites depicted as round bright dots at the marginal area of the dark hair follicle (arrow) in the en-face mode (b) and bright irregular structures at the corresponding site in the slice mode (a). $\mathbf{d}$ By conventional histology, the lengthy violet shape of the mites is visible in the hair follicle which is surrounded by an inflammatory infiltrate indicating demodex folliculitis. HE. $\times 100$. 


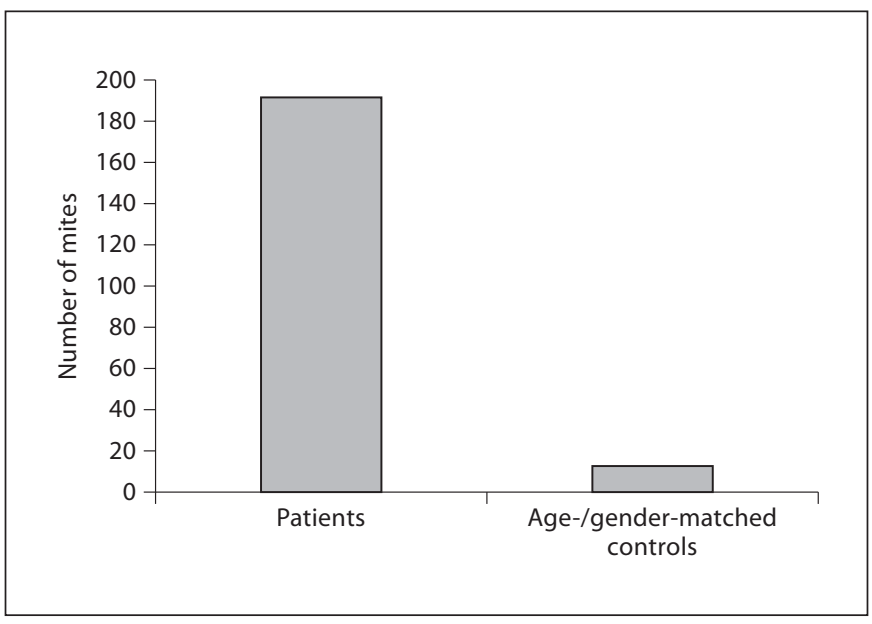

Fig. 3. The overall number of mites detected by HD-OCT in patients with skin eruptions (left column) was higher than in the age- and gender-matched control patients (right column).

Table 1. Results of HD-OCT of demodex mites in patients with facial skin manifestations, age- and gender-matched controls and a conventional skin scrape test

\begin{tabular}{llllllllr}
\hline $\begin{array}{l}\text { Pa- } \\
\text { tients }\end{array}$ & Mites/follicle & $\begin{array}{l}\text { In- } \\
\text { fested } \\
\text { folli- } \\
\text { cles }\end{array}$ & $\begin{array}{l}\text { Fol- } \\
\text { licles/ } \\
\text { field }\end{array}$ & $\begin{array}{l}\text { Skin } \\
\text { scrape } \\
\text { test }\end{array}$ & $\begin{array}{l}\text { Age-/ } \\
\text { gender- } \\
\text { matched } \\
\text { controls }\end{array}$ & $\begin{array}{l}\text { Mites/ } \\
\text { follicle }\end{array}$ & $\begin{array}{l}\text { In- } \\
\text { fested } \\
\text { folli- } \\
\text { cles }\end{array}$ & $\begin{array}{l}\text { Fol- } \\
\text { licles/ } \\
\text { field }\end{array}$ \\
\hline 1 & 5,5 & 2 & 15 & + & 1 & 0 & 0 & 14 \\
2 & 3 & 1 & 11 & + & 2 & 0 & 0 & 12 \\
3 & $5,4,3,3$ & 4 & 15 & + & 3 & 0 & 0 & 12 \\
4 & $3,4,3$ & 3 & 14 & - & 4 & 2 & 1 & 15 \\
5 & 4,2 & 2 & 12 & - & 5 & 0 & 0 & 14 \\
6 & 4,3 & 2 & 12 & + & 6 & 0 & 0 & 11 \\
7 & 3 & 1 & 15 & - & 7 & 0 & 0 & 14 \\
8 & 4 & 2 & 15 & + & 8 & 0 & 0 & 11 \\
9 & 5,5 & 2 & 16 & + & 9 & 0 & 0 & 13 \\
10 & $1,5,5$ & 3 & 16 & + & 10 & 3 & 1 & 16 \\
11 & 4,5 & 2 & 10 & + & 11 & 1 & 1 & 13 \\
12 & $4,3,2$ & 3 & 12 & + & 12 & 1 & 1 & 8 \\
13 & $5,3,3,2$ & 4 & 12 & + & 13 & 0 & 0 & 10 \\
14 & $5,4,3,2$ & 4 & 10 & + & 14 & 1 & 1 & 14 \\
15 & 3,2 & 2 & 14 & - & 15 & 0 & 0 & 11 \\
16 & 4,5 & 2 & 11 & + & 16 & 4 & 1 & 8 \\
17 & $3,2,2$ & 3 & 12 & n.p. & 17 & 0 & 0 & 9 \\
18 & $4,3,2,2$ & 4 & 10 & + & 18 & 0 & 0 & 11 \\
19 & $5,5,3,2,2$ & 5 & 12 & + & 19 & 0 & 0 & 7 \\
20 & $4,3,3,3,3,2,1$ & 7 & 12 & + & 20 & 1 & 1 & 11 \\
\hline & & & & & & & &
\end{tabular}

Follicles/field $=$ Number of all visible follicles within the field of view; infested follicles = number of follicles with demodex mites within the field of view; mites/follicle $=$ number of mites within the infested follicles; n.p. = not performed.
In the patients with demodex infestation, a mean of 3.4 mites per follicle was detected with a range of 1-5 mites per follicle. In the control patients, a mean of 0.6 (range 0-4) mites were found. In total, 192 mites were counted in the 20 patients versus 13 in the controls by HD-OCT (fig. 3). The detection rate of demodex mites by HD-OCT in infested patients was significantly higher than in healthy controls ( $p<0.0001$, Fisher's exact test).

Descriptive statistics were performed to evaluate the sensitivity and specificity for ex vivo HD-OCT images using the $\chi^{2}$ test. The statistical evaluation showed a positive predictive value of $67 \%$ (95\% CI $0.50-0.80)$ in detecting demodex mites by HD-OCT. The sensitivity was $100 \%$ because of the detection of mites in 20 out of 20 patients and the specificity was $65 \%$.

According to the manufacturer, the penetration depth of this HD-OCT system is $750 \mu \mathrm{m}$, which could not be reached in our study. However, the penetration depth was sufficient to visualize the superficial dermis of the facial skin in the en-face mode. The display of the image in the slice mode was dependent on the amount of contact gel used. With the application of a thick layer of contact gel, the skin image was located on the lower third of the screen.

There is a possibility of artifacts by motion of the skin against the device, but this was not a problem in this study as the examined skin was mostly even. However, it was recognized that the image quality was reduced in very papular skin eruptions because of the uneven surface, and the counting of follicles could only be performed when moving the cursor up and down in the en-face mode. The artefact by motion might cause a problem in the examination of elevated tumors.

\section{Skin Scrape Tests}

In all 20 patients with clinical skin manifestations, it was possible to detect demodex mites in the hair follicles by HD-OCT in the en-face mode (20/20 or $100 \%$ ), whereas the skin scrape test was not performed in 1 patient and was positive in 15 (78.9\%) and negative in 4 (21\%).

\section{Discussion}

OCT imaging has been reported before in the visualization of infestation with scabies and larva migrans [15, 20-23]. The identification of demodex mites in the hair follicle by OCT has not been reported so far.

According to the literature, demodex mites have a size of around $35 \mu \mathrm{m}$ in diameter which is hardly possible to detect in a vertical imaging mode by conventional OCT 
systems [24]. Nevertheless, the size of an adult mite can be up to $290 \mu \mathrm{m}$ in length which comes close to a scabies mite with a diameter of $240-350 \mu \mathrm{m}$, which has been successfully imaged before by OCT. In comparison to the scabies mite, the demodex mite is localized in the follicles in a vertical orientation, which makes it difficult to find in a cross-sectional image. Concerning the imaging of larva migrans by OCT, it has been reported that mainly the canals and not the larva itself could be displayed [20].

In this prospective pilot study we found the following results. First, we were able to identify demodex mites by HD-OCT in the en-face mode as bright round dots grouped at the marginal areas of the dark follicle in patients with demodex-related facial skin eruptions. Second, the comparison with age- and gender-matched controls revealed a higher rate of demodex mites in the patients. Third, there was improved detection of demodex mites by HD-OCT over the skin scrape test in the examined patients.

We propose that the detection of demodex mites by HD-OCT is mainly possible because of the horizontal imaging mode and the high resolution of $3 \mu \mathrm{m}$. As a matter of fact, the demodex mites are located in the infundibulum of the hair follicle in groups upside down of the hair bulb, which makes them better visible in a horizontal imaging mode. There are other OCT devices which are able to compose 3-dimensional images, although not in a real-time manner, so the generation of such an image might be time-consuming. Detection of demodex mites might be possible with other OCT systems, but, to our knowledge, this has not yet been performed.

In the en-face mode, the mites present as prominent bright round dots within the otherwise dark follicle, comparable to the horizontal mode of imaging used in reflectance confocal microscopy (RCM), which we and others have studied for the visualization and quantification of demodex mites in vivo [25-27]. In this study, using HDOCT, demodex mites were found to a far lesser extent in the controls; this is in contrast to our experience with $\mathrm{RCM}$, where the mites were also present in the controls with a mean number of 22.4 in the $5 \times 5 \mathrm{~mm}$ scanning area. In addition, with RCM, the approximate number of follicles in the field of view was higher than the results we obtained by HD-OCT. This indicates that the RCM technology is more accurate, possibly because of a better contrast and a higher resolution $(1.5 \mu \mathrm{m})$ which allow more follicles to be visualized.

Nevertheless, HD-OCT imaging was sufficient for the detection of demodex mites in all patients and was superior to the conventional skin scrape test which failed to recognize mites in 4 of 19 cases. In a recent study, it could be shown that the standardized skin surface biopsy is very effective in the detection of demodex mites compared to microscopic examination of secretions from sebaceous glands and might therefore be regarded as a reference standard for the detection of mites [28]. However, all of these (semi-)invasive tests have the disadvantage of a time delay before the result is obtained and cannot be repeated immediately if the result is negative whereas the detection of mites by HD-OCT is easily reproducible on different test sites and the result can be directly evaluated.

This pilot study provides, for the first time, data on the visualization and detection of demodex mites with an OCT device. It would be interesting, in further studies, to compare the HD-OCT results with the skin surface biopsy and to evaluate if the HD-OCT detection area of $1.8 \times 1.5 \mathrm{~mm}$ is sufficient when it is compared to the area of $1 \mathrm{~cm}^{2}$ which is the widely accepted standard area for the evaluation of demodex density.

Although RCM evaluation of demodex mites as published by our group is a very accurate instrument for this indication, the time (10 min per patient) and effort needed to apply RCM are significantly greater than in the HDOCT imaging with a time frame of about $2-5 \mathrm{~min}$. The new RCM hand-held device (Vivascope ${ }^{\circledR} 3000$ handheld, Mavig GmbH, Munich, Germany) provides $1 \times 1 \mathrm{~mm}$ images in a few seconds and therefore might be also an option for the fast detection of mites. HD-OCT, despite obtaining a lower resolution than RCM, may be more practicable for the diagnosis and quantification of demodex mites, while the technique of RCM with its nearly histological resolution seems better designed for more complex indications such as the differentiation between nevus and malignant melanoma $[29,30]$.

Regarding the performance of HD-OCT in this study, we observed a limitation regarding the penetration depth because $750 \mu \mathrm{m}$ could not be reached. The HD-OCT images started to get unclear at the level of the superficial dermis. As demodex mites are located at low depths $(<100 \mu \mathrm{m})$, the image quality was adequate to display the mites in this setting, but deeper mid-dermal structures could not be visualized. Second, the image localization on the computer screen depended on the amount of coupling gel applied. As an advantage, we noted the fast construction and the high quality of the en-face images.

Further studies will determine if HD-OCT could be a valuable tool in the treatment monitoring of demodexassociated skin diseases. For now, it offers a noninvasive and fast in vivo technique for the immediate diagnosis of demodex mites, which is indeed superior to the semi-invasive skin scrape test. 


\section{Acknowledgement}

This work was supported by the Curd-Bohnewand-Fonds of the University of Munich (T.M.), by the Matthias Lackas Foundation and the Dr. Helmut Legerlotz Foundation (C.B.).

\section{Disclosure Statement}

The HD-OCT device used in this study was provided by AGFA HealthCare GmbH. T.M. has received a speaker's honorarium by AGFA HealthCare GmbH.

\section{References}

1 Baima B, Sticherling M: Demodicidosis revisited. Acta Derm Venereol 2002;82:3-6.

2 Burns DA: Follicle mites and their role in disease. Clin Exp Dermatol 1992;17:152-155.

3 Borgo SN, Sattler EC, Hogardt M, Adler K, Plewig G: PCR analysis for Wolbachia in human and canine demodex mites. Arch Dermatol Res 2009;301:747-752.

4 Rufli T, Mumcuoglu Y: The hair follicle mites Demodex folliculorum and Demodex brevis: biology and medical importance. A review. Dermatologica 1981;162:1-11.

5 Rufli T, Mumcuoglu Y, Cajacob A, Buchner S: Demodex folliculorum: aetiopathogenesis and therapy of rosacea and perioral dermatitis (author's transl). Dermatologica 1981;162: $12-26$.

6 Georgala S, Katoulis AC, Kylafis GD, Koumantaki-Mathioudaki E, Georgala C, Aroni K: Increased density of Demodex folliculorum and evidence of delayed hypersensitivity reaction in subjects with papulopustular rosacea. J Eur Acad Dermatol Venereol 2001; 15:441-444.

7 Kligman AM, Christensen MS: Demodex folliculorum: requirements for understanding its role in human skin disease. J Invest Dermatol 2011;131:8-10.

8 Marks R, Dawber RP: Skin surface biopsy: an improved technique for the examination of the horny layer. Br J Dermatol 1971;84:117123.

9 Forton F, Song M: Limitations of standardized skin surface biopsy in measurement of the density of Demodex folliculorum. A case report. Br J Dermatol 1998;139:697-700.

10 Welzel J, Lankenau E, Birngruber R, Engelhardt R: Optical coherence tomography of the human skin. J Am Acad Dermatol 1997; 37:958-963.

11 Welzel J: Optical coherence tomography in dermatology: a review. Skin Res Technol 2001;7:1-9.

12 Dalimier E, Salomon D: Full-field optical coherence tomography: a new technology for $3 \mathrm{D}$ high-resolution skin imaging. Dermatology 2012;224:84-92.
13 Maier T, Braun-Falco M, Hinz T, SchmidWendtner MH, Ruzicka T, Berking C: Morphology of basal cell carcinoma in high definition optical coherence tomography: enface and slice imaging mode, and comparison with histology. J Eur Acad Dermatol Venereol 2012, E-pub ahead of print.

14 Maier T, Braun-Falco M, Laubender RP, Ruzicka T, Berking C: Actinic keratosis in the en-face and slice imaging mode of high definition optical coherence tomography and comparison with histology. Br J Dermatol 2012, E-pub ahead of print.

15 Gambichler T, Jaedicke V, Terras S: Optical coherence tomography in dermatology: technical and clinical aspects. Arch Dermatol Res 2011;303:457-473.

16 Hinz T, Ehler LK, Hornung T, Voth H, Fortmeier I, Maier T, Holler T, Schmid-Wendtner $\mathrm{MH}$ : Preoperative characterization of basal cell carcinoma comparing tumour thickness measurement by optical coherence tomography, $20-\mathrm{MHz}$ ultrasound and histopathology. Acta Derm Venereol 2012;92:132-137.

17 Cossmann M, Welzel J: Evaluation of the atrophogenic potential of different glucocorticoids using optical coherence tomography, $20-\mathrm{MHz}$ ultrasound and profilometry; a double-blind, placebo-controlled trial. $\mathrm{Br} \mathrm{J}$ Dermatol 2006; 155:700-706.

18 Hinz T, Ehler LK, Voth H, Fortmeier I, Hoeller T, Hornung T, Schmid-Wendtner $\mathrm{MH}$ : Assessment of tumor thickness in melanocytic skin lesions: comparison of optical coherence tomography, $20-\mathrm{MHz}$ ultrasound and histopathology. Dermatology 2011;223: 161-168.

19 Aydin SZ, Ash Z, Del Galdo F, Marzo-Ortega $\mathrm{H}$, Wakefield RJ, Emery P, McGonagle D: Optical coherence tomography: a new tool to assess nail disease in psoriasis? Dermatology 2011;222:311-313.

20 Morsy $\mathrm{H}$, Mogensen M, Thomsen J, Thrane L, Andersen PE, Jemec GB: Imaging of cutaneous larva migrans by optical coherence tomography. Travel Med Infect Dis 2007;5: 243-246.
21 Welzel J, Lankenau E, Birngruber R, Engelhardt R: Optical coherence tomography of the skin. Curr Probl Dermatol 1998;26:2737.

22 Mogensen M, Thrane L, Jorgensen TM, Andersen PE, Jemec GB: OCT imaging of skin cancer and other dermatological diseases. J Biophotonics 2009;2:442-451.

23 Longo C, Bassoli S, Monari P, Seidenari S, Pellacani G: Reflectance-mode confocal microscopy for the in vivo detection of Sarcoptes scabiei. Arch Dermatol 2005;141:1336.

24 Desch C: Human hair follicle mites and forensic acarology. Exp Appl Acarol 2009;49: 143-146.

25 Longo C, Pellacani G, Ricci C, De Pace B, Argenziano G, Zalaudek I: In vivo detection of Demodex folliculorum by means of confocal microscopy. Br J Dermatol 2012;166:690692.

26 Slutsky JB, Rabinovitz H, Grichnik JM, Marghoob AA: Reflectance confocal microscopic features of dermatophytes, scabies, and demodex. Arch Dermatol 2011;147:1008.

27 Sattler EC, Maier T, Hoffmann VS, Hegyi J, Ruzicka T, Berking C: Non-invasive in vivo detection and quantification of demodex mites by confocal laser scanning microscopy. Br J Dermatol 2012;167:1042-1047.

28 Askin U, Seckin D: Comparison of the two techniques for measurement of the density of Demodex folliculorum: standardized skin surface biopsy and direct microscopic examination. Br J Dermatol 2010;162:1124-1126.

29 Maier T, Sattler EC, Braun-Falco M, Korting HC, Ruzicka T, Berking C: Reflectance confocal microscopy in the diagnosis of partially and completely amelanotic melanoma: report on seven cases. J Eur Acad Dermatol Venereol 2012, E-pub ahead of print.

30 Pellacani G, Guitera P, Longo C, Avramidis $M$, Seidenari S, Menzies S: The impact of in vivo reflectance confocal microscopy for the diagnostic accuracy of melanoma and equivocal melanocytic lesions. J Invest Dermatol 2007;127:2759-2765. 